\title{
RIVAroxaban TWICE daily for lysis of thrombus in the left atrial appendage in patients with non-valvular atrial fibrillation: the RIVA-TWICE study
}

Roman Piotrowski, Beata Zaborska, Ewa Pilichowska-Paszkiet, Małgorzata Sikora-Frąc, Jakub Baran, Piotr Kułakowski

Postgraduate Medical School, Department of Cardiology, Grochowski Hospital, Warsaw, Poland

Submitted: 13 October 2018

Accepted: 22 February 2019

Arch Med Sci 2020; 16 (2): 289-296

DOI: https://doi.org/10.5114/aoms.2019.86616

Copyright (C) 2019 Termedia \& Banach

\section{Abstract}

Introduction: Rivaroxaban is a direct factor Xa inhibitor used once a day for prevention of thrombotic events in patients with atrial fibrillation (AF). However, in a small proportion of subjects thrombus in the left atrial appendage (LAA) is present despite this treatment. The aim of this study was assess the efficacy of increased dose of rivaroxaban (15 mg twice daily) treatment for lysis of thrombus in the LAA.

Material and methods: In the RIVA-TWICE prospective, open label study, with non-blinded patients and blinded outcome assessors, rivaroxaban $15 \mathrm{mg}$ twice daily for 8 weeks was administered in patients with AF who had LAA thrombus despite standard $20 \mathrm{mg}$ once a day therapy. Transesophageal echocardiography was performed at baseline and after 8 weeks. Blood samples were taken to measure the activity of the anti-Xa factor.

Results: Fifteen patients ( 9 males, mean age: $63 \pm 10$ years) were enrolled. Following 8 weeks of rivaroxaban $15 \mathrm{mg}$ twice daily, complete resolution of thrombus in the LAA was observed in 7 (46.7\%) patients. The mean activity of anti-Xa factor was significantly higher during rivaroxaban twice daily therapy compared with the standard dose. However, there were no significant differences between effectively and non-effectively treated patients. Conclusions: Rivaroxaban 15 mg twice daily seems to be safe and may dissolve LAA thrombus when standard rivaroxaban therapy is ineffective. Lower $\mathrm{CHA}_{2} \mathrm{DS}_{2}$-VASC and HAS-BLED as well as preserved LAA emptying function identified responders.

Key words: rivaroxaban, twice daily, atrial fibrillation, thrombus, left atrial appendage.

\section{Introduction}

Atrial fibrillation (AF) is the most common cardiac arrhythmia and is associated with increased risk of thromboembolic events. Consequently, patients with AF have five-fold increased risk of stroke [1]. The most common place for thrombus formation is the left atrial appendage (LAA), which is the dominant source of embolism in more than $90 \%$ of patients with AF and intracardiac thrombus [2].

Transesophageal echocardiography (TEE) remains a gold standard for assessment of the LAA [1]. When thrombus is detected, current European

\author{
Corresponding author: \\ Jakub Baran MD, PhD \\ Postgraduate Medical School \\ Department of Cardiology \\ Grochowski Hospital \\ 51/59 Grenadierow St \\ 04-073 Warsaw, Poland \\ Phone: +48 225152757 \\ E-mail: jakub.baran1111@ \\ gmail.com
}


Society of Cardiology (ESC) guidelines recommend treatment with non-antagonist vitamin $\mathrm{K}$ (NOAC) or vitamin $\mathrm{K}$ antagonist (VKA) for at least 3 weeks and to repeat TEE to ensure the absence of thrombus before performing such interventions as cardioversion or ablation [1]. Owing to easier dosing and at least the same effectiveness and a higher safety profile, NOAC are preferred over VKA for prevention of thromboembolic events in patients with non-valvular AF [1].

Rivaroxaban is a direct factor Xa inhibitor which is used for prevention of thromboembolic events in patients with nonvalvular AF or venous thrombo-embolism (VTE). In patients with AF, a single daily dose of rivaroxaban is recommended, but in a small proportion of subjects a thrombus in the LAA is present despite this treatment [3-5]. In acute pulmonary embolism (APE) or deep vein thrombosis (DVT) a twice daily regimen of rivaroxaban is recommended for the first 3 weeks, followed by once a day therapy [6]. Considering the relatively short half-life of rivaroxaban (7-11 h) [7] and rare but known cases of thrombus persistence in the LAA despite the use of rivaroxaban once a day [3-5], the question arises whether some patients with AF should receive the drug twice a day.

The present study was designed to address this issue.

\section{Material and methods}

\section{Study design and patient population}

The study protocol was approved by the Local Ethics Committee of the Postgraduate Medical School, Warsaw, Poland (No. 49/PB/2015). Informed consent was obtained from all individual participants included in the study. All procedures performed in studies involving human participants were in accordance with the ethical standards of the institutional and/or national research committee and with the 1964 Helsinki declaration and its later amendments or comparable ethical standards.

The RIVA-TWICE was a prospective, open label study, with non-blinded patients and blinded assessors, which assessed the safety and efficacy of rivaroxaban $15 \mathrm{mg}$ twice daily for 8 weeks administered for lysis of thrombus in the LAA in patients with non-valvular AF who had LAA thrombus despite standard $20 \mathrm{mg}$ once a day rivaroxaban therapy. TEE was performed at baseline and after 8 weeks of intensified rivaroxaban therapy.

The inclusion criteria were presence of thrombus in the LAA during rivaroxaban once a day therapy, age $\leq 80$ years old, lack of advanced renal failure (creatinine clearance $<30 \mathrm{ml} / \mathrm{min} / 1.73 \mathrm{~m}^{2}$ calculated using Cockcroft and Gault formula), no significant liver dysfunction (alanine transami- nase level exceeding three times the upper normal limit) and no history of major bleeding, including intracranial hemorrhage. Women of childbearing potential were excluded unless an effective contraception was documented.

Every effort was made to explain to the patient the importance of not omitting any dose of rivaroxaban. All patients were encouraged to take regularly, morning and evening, a dose of rivaroxaban with a meal, but no formal questionnaire was used to assess adherence.

\section{Echocardiography}

All echocardiographic studies were performed using the Vivid 9 ultrasound system (GE Medical System, Horten, Norway, 2010). Transthoracic echocardiographic examinations (TTE) were performed and measurements were made in accordance with the recommendations for cardiac chamber quantification by echocardiography in adults [8]. The LA diameter (LAd) was measured at end-systole in the parasternal long-axis (PLAX) view. The left ventricular (LV) diameter (LVEDd) was measured at end-diastole in the PLAX view. The LA volume (LAV) was calculated from the apical 4-chamber (4C) and 2-chamber (2C) views using the biplane area-length method. The LAV index (LAVi) was defined as LA volume divided by the body surface area (BSA). The LV ejection fraction (LVEF) was calculated using Simpson's method.

Transesophageal echocardiography examinations were performed according to the standard practice European Association of Echocardiography (EAE) guidelines $[9,10]$ with $6 \mathrm{~T}$ and $6 \mathrm{TC}$ multiplane TEE probes. Loops of 3 cardiac cycles were stored digitally and analyzed offline with dedicated software (EchoPac, GE Healthcare) by two independent experienced echocardiographers blinded to demographic and clinical data, TEE timing and previous echocardiographic results. All images were randomly numbered and the patient's personal data were deleted before analysis. The images were meticulously evaluated for the presence or absence of LAA thrombi and the spontaneous echo contrast (SEC) phenomenon. Echocardiographic images of the LAA were assessed twice (after completion of enrollment and 3 months later during the final analysis) in order to ensure reproducibility of detection of LAA thrombus. Reproducibility of echocardiographic detection of LAA thrombus was $100 \%$.

Thrombus was defined as an echo-dense mass attached to the LAA wall or filling out the LAA cavity with or without partial protrusion into the LA cavity. The area of thrombus was measured indirectly and expressed as a percentage of the LAA area. Spontaneous echo contrast was defined as dynamic "smoke-like" echoes swirling in the LA 
or LAA. The severity of SEC was graded from 0 to $4+$ as described previously [11]. Interobserver differences in the grading of SEC were resolved by consensus. Spontaneous echo contrast was not considered as a thrombus. The LAA velocity was recorded by placing the pulse wave (PW) Doppler gate within $1 \mathrm{~cm}$ of the LAA orifice.

\section{Measurements of activity of anti-Xa factor}

Anticoagulant effects of rivaroxaban were assessed using the anti-factor Xa chromogenic method (Diagnostica Stago, Asnières-sur-Seine, France). The activity of the anti-Xa factor was measured during once a day and after 8 weeks of twice daily rivaroxaban therapy. The therapeutic range of anti-Xa activity is between 20 and 500

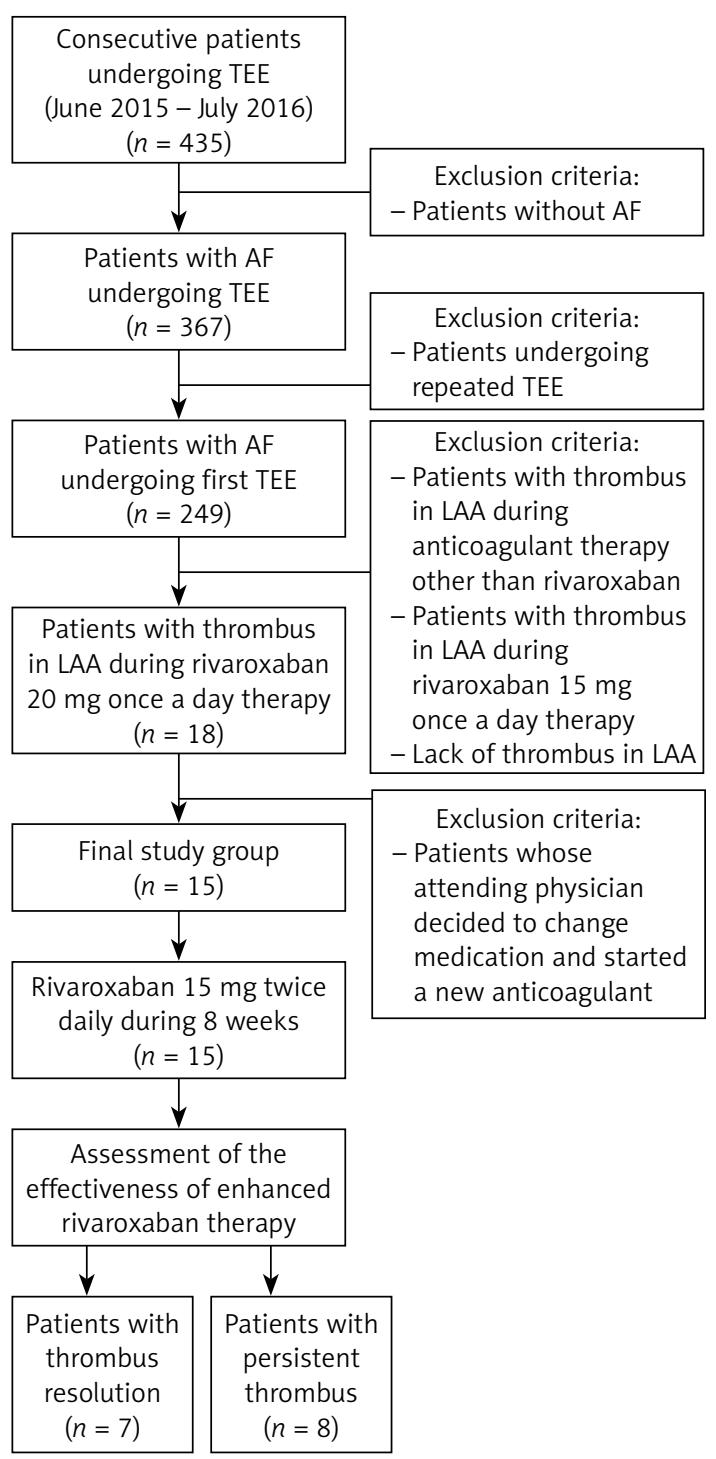

Figure 1. Flow diagram of patient recruitment and progress throughout the study. Of 435 patients who underwent TEE, 15 patients finally entered the RIVA-TWICE study

TEE - transesophageal echocardiography, AF-atrial fibrillation, LAA - left atrial appendage. $\mathrm{ng} / \mathrm{ml}$. The anti-Xa activity was measured 3, 12 and $24 \mathrm{~h}$ after the morning dose of rivaroxaban, both during $20 \mathrm{mg}$ once a day rivaroxaban therapy and after switching to $15 \mathrm{mg}$ twice a day.

\section{Bleeding complications}

All patients were instructed to report immediately any bleeding complications which were classified using the BARC scale [12].

\section{Statistical analysis}

The results are presented as mean \pm SD or numbers and percentages. Numerical variables were compared using a 2-sided paired t-test. A $p$-value $<0.05$ was considered significant.

\section{Results}

Between June 2015 and July 2016, 435 patients underwent TEE in our echocardiography laboratory. Of them, 367 TEE were performed in subjects with AF. Out of this group in 249 patients this was the first TEE performed during the study period (we excluded all repeated TEE). Of these 249 patients, 18 subjects who had LAA thrombus on a standard dose of rivaroxaban were candidates to enter the study; however, in three of them the attending physician decided to change the medication and started a new anticoagulant. Thus, the final study group consisted of 15 patients (9 males, mean age: $63 \pm 10$ years). The patient

Table I. Demographic, clinical and echocardiographic characteristics of subjects enrolled in the RIVATWICE study

\begin{tabular}{|lc|}
\hline Parameter & Results \\
\hline No of patients & 15 \\
\hline Age [years] & $63 \pm 10$ \\
\hline Male gender, $n(\%)$ & $9(60)$ \\
\hline Height [m] & $1.66 \pm 0.09$ \\
\hline Weight [kg] & $87 \pm 16.2$ \\
\hline BMI [kg/m²] & $31.21 \pm 3.55$ \\
\hline CHA DS $_{2}$-VASc & $3.2 \pm 1.7$ \\
\hline HAS-BLED & $1.3 \pm 0.9$ \\
\hline Echocardiographic parameters: & \\
\hline LVEF $(\%)$ & $49 \pm 11$ \\
\hline LVEdd [mm] & $49 \pm 6$ \\
\hline LAd [mm] & $43 \pm 5$ \\
\hline LAV [ml] & $117 \pm 35$ \\
\hline LAVi [ml/m²] & $59 \pm 16$ \\
\hline LAA V [m/s] & $0.22 \pm 0.08$ \\
\hline
\end{tabular}

LVEF - left ventricular ejection fraction, LVEdd - left ventricular diastolic diameter, LAd - left atrial diameter, LAV - left atrial volume, LAVi - left atrial volume index, LAA V-emptying velocity from $L A A$. Results are presented as mean $\pm S D$ or numbers and percentages. 
flow diagram is shown in Figure 1. Five patients underwent TEE before planned catheter ablation of $\mathrm{AF}$ and the remaining 10 patients before elective cardioversion of AF. Demographic, clinical and echocardiographic characteristics of the studied population are presented in Table I.

All patients completed follow-up. Drug compliance was achieved in all patients - none of them returned unused pills at the final visit. Complete resolution of thrombus in the LAA was observed in $7(46.7 \%)$ patients. Comparison of demographic, clinical and echocardiographic parameters between patients with thrombus resolution and those in whom thrombus persisted are presented in Table II. Patients in whom thrombus was dissolved had a significantly lower $\mathrm{CHA}_{2} \mathrm{DS}_{2}$-VASc score (2.3 \pm 1.5 vs. $4.0 \pm 1.5, p=0.0463)$, HAS-BLED score ( 0.7 \pm 0.8 vs. $1.8 \pm 0.7, p=0.0168)$ and higher mean LAA emptying velocity $(0.27 \pm 0.09$ vs. $0.18 \pm 0.02 \mathrm{~m} / \mathrm{s}$, $p=0.0198$ ). Duration of prior anticoagulation with rivaroxaban $20 \mathrm{mg}$ once a day was similar in both groups. No association between thrombus area and thrombus resolution was observed (Table II).

In all patients the anti-Xa activity was higher during rivaroxaban twice daily than once a day therapy, especially 12 and $24 \mathrm{~h}$ after the first dose (Figure 2). There were no significant differences in the mean values of anti-Xa factor activity between effectively and non-effectively treated patients (Figure 3). Individual values are presented in Figure 4. None of the patients had any bleeding event - the BARC scale score in all patients was 0. Examples of original TEE images before and after enhanced rivaroxaban therapy are shown in Figure 5.

\section{Discussion}

To our knowledge, this is the first study describing the efficacy of rivaroxaban twice daily therapy for lysis of thrombus in the LAA in patients with non-valvular AF treated with a standard once a day dose of rivaroxaban.

Table II. Comparison of demographic and clinical characteristics between patients in whom thrombus persisted despite rivaroxaban twice daily therapy and patients in whom thrombus was dissolved

\begin{tabular}{|c|c|c|c|}
\hline Parameter & $\begin{array}{l}\text { Patients with persisting } \\
\text { thrombus }(n=8)\end{array}$ & $\begin{array}{l}\text { Patients in whom thrombus } \\
\text { was dissolved }(n=7)\end{array}$ & $P$-value \\
\hline Age [years] & $64 \pm 10$ & $62 \pm 8$ & NS \\
\hline Male, $n(\%)$ & $4(50)$ & $5(71)$ & NS \\
\hline Height $[\mathrm{m}]$ & $1.66 \pm 0.09$ & $1.70 \pm 0.13$ & NS \\
\hline Weight $[\mathrm{kg}]$ & $87 \pm 16.1$ & $87 \pm 19.1$ & NS \\
\hline $\mathrm{BMI}\left[\mathrm{kg} / \mathrm{m}^{2}\right]$ & $31.21 \pm 3.55$ & $29.67 \pm 3.36$ & NS \\
\hline $\mathrm{CHA}_{2} \mathrm{DS}_{2}-\mathrm{VASC}$ & $4.0 \pm 1.5$ & $2.3 \pm 1.5$ & 0.0463 \\
\hline HAS-BLED & $1.8 \pm 0.7$ & $0.7 \pm 0.8$ & 0.0168 \\
\hline $\begin{array}{l}\text { Duration of prior } \\
\text { anticoagulation with } \\
\text { rivaroxaban } 20 \mathrm{mg} \text { once a day } \\
\text { [weeks] }\end{array}$ & $20 \pm 16$ & $26 \pm 17$ & NS \\
\hline $\begin{array}{l}\text { Thrombus area before } \\
\text { rivaroxaban twice daily }\left[\mathrm{cm}^{2}\right]\end{array}$ & $1.73 \pm 1.51$ & $2.98 \pm 1.12$ & NS \\
\hline $\begin{array}{l}\text { LAA area before rivaroxaban } \\
\text { twice daily }\left[\mathrm{cm}^{2}\right]\end{array}$ & $6.29 \pm 1.92$ & $6.07 \pm 1$ & NS \\
\hline Antiplatelet agents & 0 & 1 & NS \\
\hline \multicolumn{4}{|l|}{ Echocardiographic parameters: } \\
\hline LVEF (\%) & $49 \pm 13$ & $50 \pm 9$ & NS \\
\hline LVEdd [mm] & $50 \pm 7$ & $48 \pm 5$ & NS \\
\hline LAd $[\mathrm{mm}]$ & $43 \pm 7$ & $43 \pm 3$ & NS \\
\hline LAV $[\mathrm{ml}]$ & $125 \pm 41$ & $106 \pm 25$ & NS \\
\hline $\operatorname{LAVi}\left[\mathrm{ml} / \mathrm{m}^{2}\right]$ & $63 \pm 19$ & $54 \pm 11$ & NS \\
\hline LAA $\vee[\mathrm{m} / \mathrm{s}]$ & $0.18 \pm 0.02$ & $0.27 \pm 0.09$ & 0.0198 \\
\hline
\end{tabular}

LVEF - left ventricular ejection fraction, LVEdd - left ventricular diastolic diameter, LAd - left atrial diameter, LAV - left atrial volume, $L A V i$ - left atrial volume index, LAA V-emptying velocity from $L A A$. 


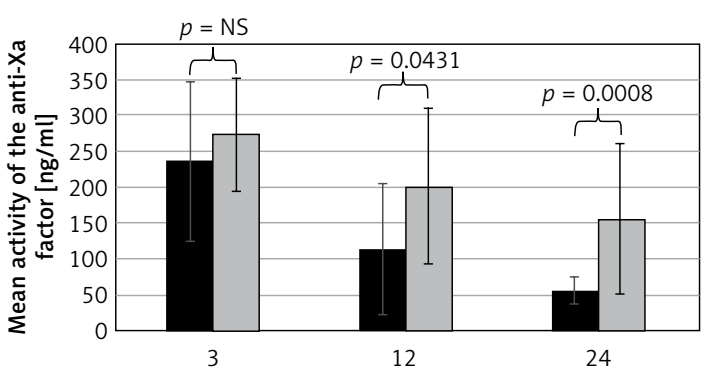

Time point measurement after morning dose of rivaroxaban $[\mathrm{h}]$

Figure 2. Comparison of activity of the anti-Xa factor at 3, 12 and $24 \mathrm{~h}$ after morning dose of rivaroxaban between once a day (black bars) and twice daily (grey bars) rivaroxaban therapy

A

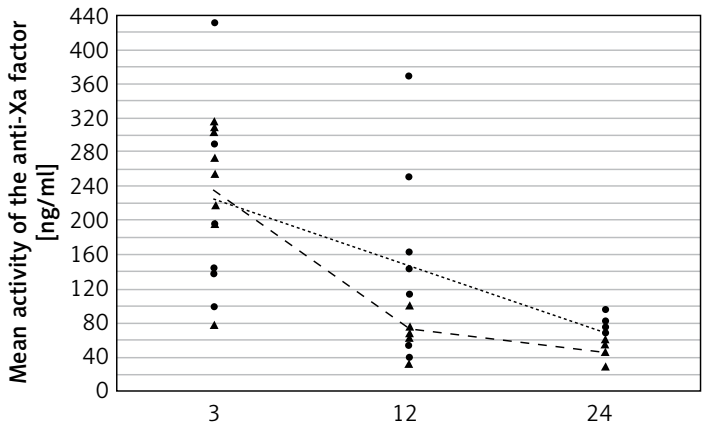

Time point measurement after morning dose of rivaroxaban [h]

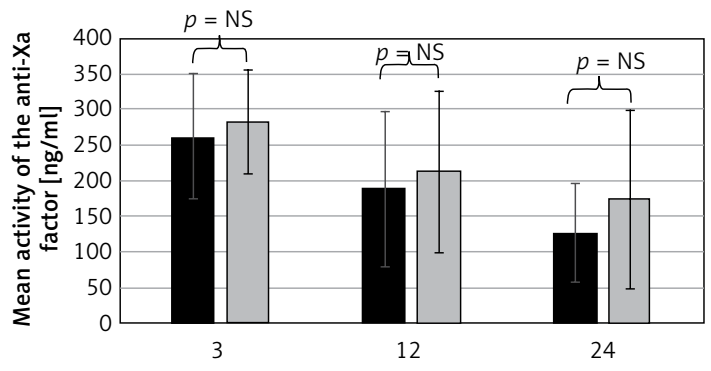

Time point measurement after morning dose of rivaroxaban [h]

Figure 3. Comparison of the mean activity of the anti-Xa factor at 3, 12 and $24 \mathrm{~h}$ after morning dose of rivaroxaban between group with thrombus resolution (black bars) and with persisting thrombus (grey bars) during twice daily rivaroxaban therapy

\section{B}

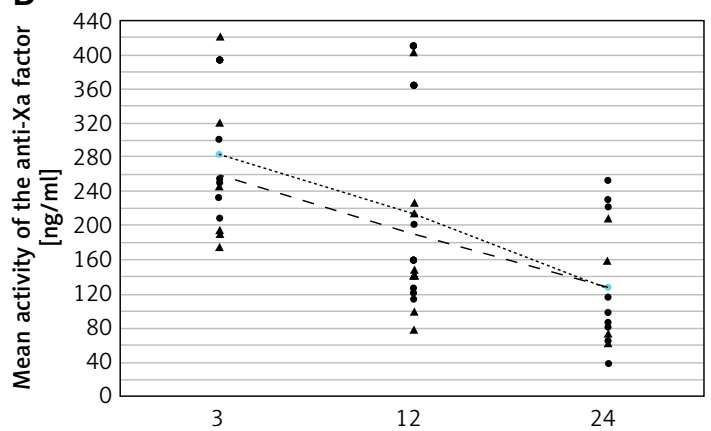

Time point measurement after morning dose of rivaroxaban [h]

$$
\text { - Thr (+) \ Thr (-) }
$$

Figure 4. Individual values of anti-Xa factor activity at baseline (during once a day dosing) (A) and after increased dose of rivaroxaban (twice a day) (B). Dashed line shows the mean values of anti-Xa factor activity in patients with thrombus resolution and dotted line shows the mean values of anti-Xa factor activity in patients with persisting thrombus

Thr (+) - no thrombus resolution, Thr (-) - thrombus resolution
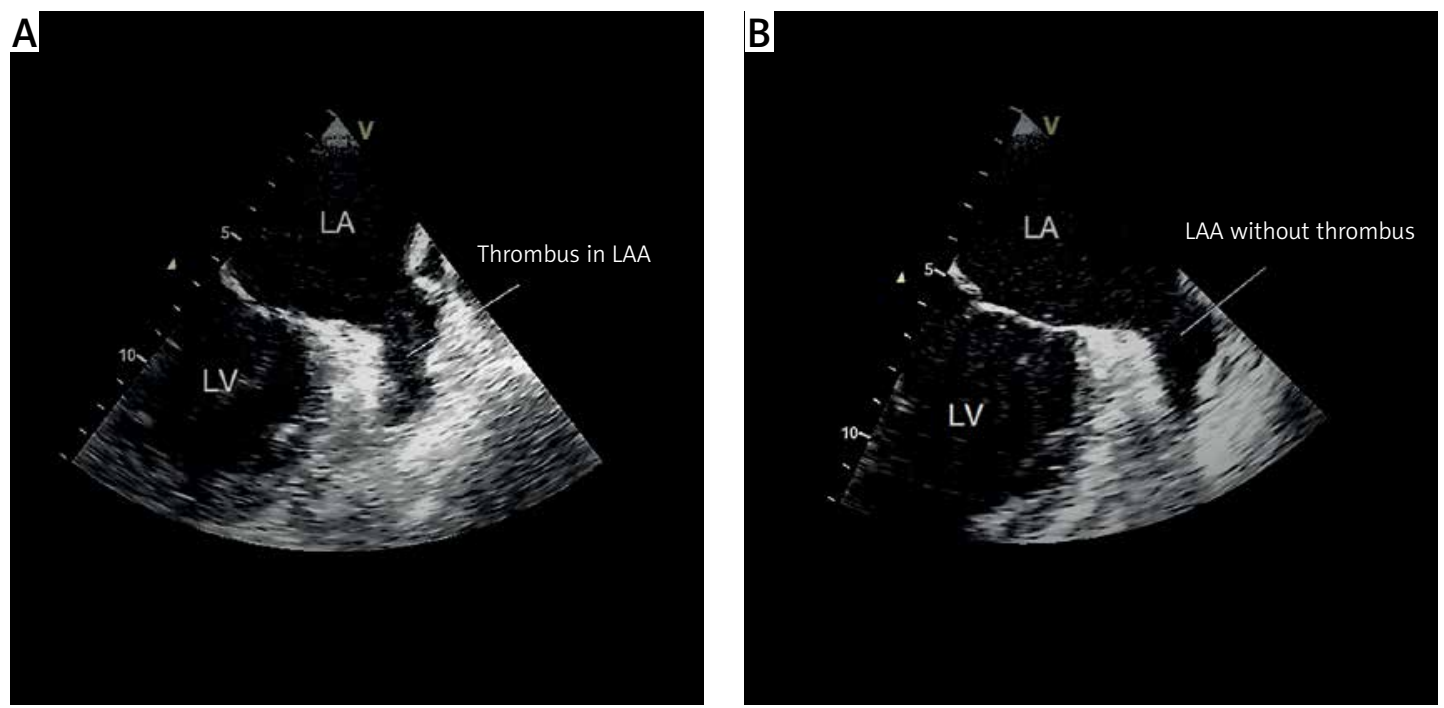

Figure 5. Examples of original TEE images before $(\mathbf{A})$ and after (B) enhanced rivaroxaban therapy

$L A$ - left atrium, $L V$ - left ventricle, $L A A-$ left atrial appendage.

Despite the fact that NOAC are effective and safe in preventing thrombo-embolic complications in patients with $A F$, their protective effect is not
$100 \%$ and stroke or other peripheral thromboembolism may occur in $0.51 \%$ to $2.67 \%$ of patients on rivaroxaban therapy $[3,13]$. This may be due to 
the fact that in a small proportion of patients (up to $4.1 \%)$, a thrombus in the LAA may persist despite therapy with standard doses of NOAC [3-5]. In these patients the risk of thromboembolic events is high and alternative therapies are needed. These usually consist of increasing the dose of anticoagulant or changing to another agent, usually with a different mechanism of action.

To date, a total of 14 studies [14-16] have reported the use of all types of NOAC, including rivaroxaban, for the resolution of LAA thrombus in patients with non-valvular AF in whom previous anticoagulation was ineffective. These studies generally indicated beneficial results - the change of anticoagulant agent resulted in resolution of thrombus. However, most of these studied are case reports and only standard doses of anticoagulants were used [15, 16]. Three case reports demonstrated resolution of thrombus in the LAA during rivaroxaban once a day therapy. Two case reports showed a beneficial effect during dabigatran twice daily therapy, and seven case reports showed resolution of thrombus in the LAA during apixaban twice a day therapy.

The only prospective, multicenter study was the X-TRA trial, which showed that rivaroxaban once a day is effective in dissolving LAA thrombus in patients with non-valvular AF and suboptimal VKA therapy [14]. In the X-TRA study rivaroxaban was used for 6 weeks once a day and the thrombus resolution rate was $41.5 \%$, whereas resolution or reduction in thrombus volume was evident in $60.4 \%$ of patients. However, in the X-TRA study a single dose of rivaroxaban was used, whereas our study aimed to assess thrombus resolution when a single dose of rivaroxaban was ineffective and the rivaroxaban dose was increased to $15 \mathrm{mg}$ twice daily. There are no data from prospective studies with other NOAC, although one prospective study with dabigatran is still ongoing [17].

The drawback of an increased dose of rivaroxaban is that in non-responders the risk of bleeding may be unnecessarily increased; therefore an assessment of the potential benefits and the risk of bleeding is necessary.

In another study Hammerstingl et al. compared the effects of VKA vs. NOAC if LAA thrombus was confirmed during VKA therapy [18]. The resolution rates of thrombus were $75 \%$ for rivaroxaban and $60 \%$ for dabigatran and apixaban whereas for VKA the resolution rate was only $8.1 \%$. However, again that study differed from ours because the patients had persistent thrombus despite VKA therapy and not during rivaroxaban treatment as in our study. In another report, Watanabe et al. [19] demonstrated in 2 patients efficacy of dabigatran when rivaroxaban therapy was ineffective. However, there are no data for increased dose for rivarox- aban if LAA thrombus is still present despite standard but ineffective rivaroxaban therapy, and thus comparing these studies with ours is difficult. To confirm our preliminary suggestions a randomized study is needed.

Rivaroxaban has a relatively short half-life of 7-11 $\mathrm{h}$ and may not be effective in some patients with AF. The once a day dosing of rivaroxaban, proposed for prevention of stroke in patients with AF, was developed from a population pharmacokinetic model derived from the studies of patients treated from DVT [20]. The DVT population data were modified to reflect demographic characteristics of patients with AF; however, such an approach may be less accurate than the usually performed dosing study in the target population, in this case patients with AF. Patients with DVT, despite statistical simulations and adjustments, may still differ from patients with AF and the results obtained in the DVT population may not necessarily be extrapolated to AF patients. In addition, one recent meta-analysis showed that the twice daily dosing regimen of NOAC offers better brain protection, defined as prevention of ischemic stroke, in patients with AF than once a day therapy [21]. However, the main limitations of this meta-analysis are differences between study populations and only indirect comparisons of the values of hazard ratios of individual NOAC versus warfarin. Only head-to-head comparison between the once a day vs twice daily dosing regimen of a given NOAC would allow us to establish which dosage regimen is better. Finally, although there are suggestions that mechanisms of clot formation in DVT are similar to those for fibrillating atrium and non-contracting LAA [22], it may be speculated that arterial LAA thrombi and venous thrombi may have different compositions, which may have an impact on NOAC efficacy. Clot dissolution occurred during enhanced rivaroxaban therapy, so it is most likely that the drug itself caused thrombus lysis. However, other endogenous mechanisms may also be responsible for this effect.

Our study showed that patients treated with an increased dose of rivaroxaban had significantly higher anti-Xa activity than during standard once a day therapy, especially 12 and $24 \mathrm{~h}$ after the first dose. This may suggest that indeed once a day therapy does not ensure adequate anti-Xa activity throughout $24 \mathrm{~h}$. However, there was no difference in anti-Xa activity between responders and non-responders to enhanced rivaroxaban therapy. This may be due to multiple factors influencing clot formation in the LAA such as LAA morphology, LAA emptying velocity, other procoagulant pathways or prothrombotic factors. Thus, the anti-Xa activity measured in peripheral blood may not be an optimal parameter to predict antithrombotic effi- 
cacy of rivaroxaban. The fact that in our study responders to the increased dose of rivaroxaban had higher emptying velocity of LAA and a lower number of procoagulant risk factors included in the $\mathrm{CHA}_{2} \mathrm{DS}_{2}$-VASC and HAS-BLED scores is in line with the multifactorial nature of clot formation in the LAA, possibly including also the shape of the LAA.

The $\mathrm{CHA}_{2} \mathrm{DS}_{2}$-VASC and HAS-BLED scales show risk of stroke and bleeding, respectively, but these scales are also an indicator of general health. Poor general condition may lead to abnormalities in the coagulation and fibrinolytic pathways which may be associated with increased risk of thrombus formation and stroke as a final effect. Thus, the patients in a better condition, expressed as lower $\mathrm{CHA}_{2} \mathrm{DS}_{2}$-VASc and HAS-BLED scores, may have a lower tendency to thrombus formation, lower activation of procoagulant factors and higher probability of thrombus resolution during intensified anticoagulant therapy.

It may be speculated that patients with reduced emptying velocity of LAA require more intensive anticoagulant therapy. However, a prospective, randomized trial is needed to confirm our preliminary suggestion.

On the other hand, it may be speculated that patients treated with once a day rivaroxaban therapy may develop or sustain LAA thrombus because of subtherapeutic drug levels. In the literature, rivaroxaban peak concentrations documented in clinical trials are in the range $40-250 \mathrm{ng} / \mathrm{ml}$. As shown in Figure 4, in none of our patients peak was baseline anti-Xa activity below $40 \mathrm{ng} / \mathrm{ml}$. However, in 2 patients during once a day rivaroxaban therapy, anti-Xa activity measured 12 and $24 \mathrm{~h}$ after rivaroxaban intake was below $40 \mathrm{ng} / \mathrm{ml}$. This may suggest that in real life in some patients rivaroxaban concentrations may be suboptimal several hours after drug consumption [23]. It has been shown that there is relatively high variability in the concentration of NOAC [23]. Moreover, variability was higher in patients treated with the lowest dose of NOAC. On average, the drug concentration levels varied nearly 15 -fold among the patients for rivaroxaban. This fact may have very important practical implications, because the use of the smallest effective dose is usually beneficial, but this may result in greater variability of drug concentration. This may lead to time windows in which anticoagulation could be ineffective and thrombus formation may occur. In these patients a twice daily regimen could help reduce the variability of the plasma drug concentration and provide a better anticoagulant effect. In our patients the variability of the concentration of the drug was higher during the $20 \mathrm{mg}$ once a day than $15 \mathrm{mg}$ twice daily regimen (Figure 4). Switching to twice a day decreases the variability and combined with better general condition could help in thrombus resolution.

This study has several limitations. Firstly, because the study is small, it is only hypothesis-generating. Secondly, there was no control group consisting of patients who continued the once a day regimen; thus, the impact of duration of NOAC therapy on LAA thrombus was not controlled. However, randomization to continuation of so far ineffective anticoagulant may be questionable and duration of anticoagulant therapy before inclusion in the study was similar in patients in whom LAA thrombus disappeared versus those with persistent thrombus. Thirdly, although all patients were instructed to take regularly twice daily pills with rivaroxaban and none of them returned unused pills at the end of the study, no formal questionnaire was used to assess adherence. Fourthly, this approach was not compared with another anticoagulant drug. Fifthly, duration of rivaroxaban twice daily therapy (8 weeks) was chosen arbitrarily. Finally, TEE has well-known limitations in detecting LAA thrombus because it may be subjective and operator-dependent. However, we did our best to overcome these limitations by blinded and repeated TEE image assessment by two experienced echocardiographers.

In conclusion, rivaroxaban administered $15 \mathrm{mg}$ twice daily seems to be safe and may dissolve LAA thrombus. Lower $\mathrm{CHA}_{2} \mathrm{DS}_{2}-\mathrm{VASC}$ and HAS-BLED scores as well as preserved LAA emptying function identify responders whereas mean anti-Xa factor activity does not. Further studies are needed to confirm these preliminary findings.

\section{Acknowledgments}

This study was funded by a research grant from the Postgraduate Medical School, Warsaw, Poland, No. 501-1-1-10-14-15/16.

\section{Conflict of interest}

The authors declare no conflict of interest.

\section{References}

1. Kirchhof P, Benussi S, Kotecha D, et al. 2016 ESC Guidelines for the management of atrial fibrillation developed in collaboration with EACTS. Europace 2016; 18: 1609-78.

2. Watson T, Shantsila E, Lip GY. Mechanisms of thrombogenesis in atrial fibrillation: Virchow's triad revisited. Lancet 2009; 373: 155-66.

3. Cappato R, Ezekowitz MD, Klein AL, et al. Rivaroxaban vs. vitamin $\mathrm{K}$ antagonists for cardioversion in atrial fibrillation. Eur Heart J 2014; 35: 3346-55.

4. Zylla MM, Pohlmeier M, Hess A, et al. Prevalence of intracardiac thrombi under phenprocoumon, direct oral anticoagulants (dabigatran and rivaroxaban), and bridging therapy in patients with atrial fibrillation and flutter. Am J Cardiol 2015; 115: 635-40. 
5. Sikorska A, Baran J, Pilichowska-Paszkiet E, et al. Risk of left atrial appendage thrombus in patients scheduled for ablation for atrial fibrillation: beyond the CHA2DS2VASc score. Pol Arch Med Wewn 2015; 125: 921-8.

6. Konstantinides SV, Torbicki A, Agnelli G, et al. 2014 ESC guidelines on the diagnosis and management of acute pulmonary embolism. Eur Heart J 2014; 35: 3033-69, 3069a-69k.

7. Steffel J, Verhamme P, Potpara TS, et al. The 2018 European Heart Rhythm Association Practical Guide on the use of non-vitamin $\mathrm{K}$ antagonist oral anticoagulants in patients with atrial fibrillation. Eur Heart J 2018; 39: 1330-93.

8. Lang RM, Badano LP, Mor-Avi V, et al. Recommendations for cardiac chamber quantification by echocardiography in adults: an update from the American Society of Echocardiography and the European Association of Cardiovascular Imaging. J Am Soc Echocardiogr 2015; 28: 1-39.

9. Pepi M, Evangelista A, Nihoyannopoulos P, et al. Recommendations for echocardiography use in the diagnosis and management of cardiac sources of embolism: European Association of Echocardiography (EAE) (a registered branch of the ESC). Eur J Echocardiogr 2010; 11: 461-76.

10. Flachskampf FA, Badano L, Daniel WG, et al. Recommendations for transoesophageal echocardiography: update 2010. Eur J Echocardiogr 2010; 11: 557-76.

11. Fatkin D, Kelly RP, Feneley MP. Relations between left atrial appendage blood flow velocity, spontaneous echocardiographic contrast and thromboembolic risk in vivo. J Am Coll Cardiol 1994; 23: 961-9.

12. Mehran R, Rao SV, Bhatt DL, et al. Standardized bleeding definitions for cardiovascular clinical trials: a consensus report from the Bleeding Academic Research Consortium. Circulation 2011; 123: 2736-47.

13. Patel MR, Mahaffey KW, Garg J, et al. Rivaroxaban versus warfarin in nonvalvular atrial fibrillation. $N$ Engl J Med 2011; 365: 883-91.

14. Lip GY, Hammerstingl C, Marin F, et al. Left atrial thrombus resolution in atrial fibrillation or flutter: results of a prospective study with rivaroxaban (X-TRA) and a retrospective observational registry providing baseline data (CLOT-AF). Am Heart J 2016; 178: 126-34.

15. Li Y, Lin J, Peng C. Resolution of massive left atrial appendage thrombi with rivaroxaban before balloon mitral commissurotomy in severe mitral stenosis: a case report and literature review. Medicine 2016; 95: e5577.

16. Piotrowski R, Zaborska B, Baran J, Sikora-Frąc M, Kułakowski P. Rivaroxaban twice daily for lysis of left atrial appendage thrombus: a potential new therapeutic option. Pol Arch Med Wewn 2016; 126: 430-1.

17. Ferner M, Wachtlin D, Konrad T, et al. Rationale and design of the RE-LATED AFAFNET 7 trial: REsolution of Left atrial-Appendage Thrombus - effects of Dabigatran in patients with Atrial Fibrillation. Clin Res Cardiol 2016; 105: 29-36.

18. Hammerstingl C, Nickenig G, Weber M, Lambers M. Direct acting oral anticoagulants are more effective than vitamin-K-antagonists for the resolution of established left atrial thrombi in patients with atrial fibrillation. Eur Heart J 2015; 65: 849-1187.

19. Watanabe T, Shinoda Y, Ikeoka K, et al. Dabigatran therapy resulting in the resolution of rivaroxaban-resistant left atrial appendage thrombi in patients with atrial fibrillation. Intern Med 2017; 56: 1977-80.

20. Mueck W, Lensing AW, Agnelli G, Decousus H, Prandoni P, Misselwitz F. Rivaroxaban: population pharmaco- kinetic analyses in patients treated for acute deep-vein thrombosis and exposure simulations in patients with atrial fibrillation treated for stroke prevention. Clin Pharmacokinet 2011; 50: 675-86.

21. Clemens A, Noack H, Brueckmann M, Lip GY. Twice- or once-daily dosing of novel oral anticoagulants for stroke prevention: a fixed-effects meta-analysis with predefined heterogeneity quality criteria. PLoS One 2014; 9: e99276.

22. Ageno W, Dentali F. Venous thromboembolism and arterial thromboembolism. Many similarities, far beyond thrombosis per se. Thromb Haemost 2008; 100: 181-3.

23. Testa S, Tripodi A, Legnani C, et al. Plasma levels of di rect oral anticoagulants in real life patients with atrial fibrillation: results observed in four anticoagulation clinics. Thromb Res 2016; 137: 178-83. 